\title{
Dynamic Games with Incomplete Knowledge in Metric Spaces
}

\author{
I.V. KONNOV ${ }^{1}$
}

\begin{abstract}
We describe a model of a discrete time dynamic system with active elements (players). All the system states are contained in a set of a metric space. Each state is associated with the common system utility value and player shares. Feasible coalitions of players can change the system state, but each move requires certain expenses. We suppose that the players may have only restricted and local knowledge about the system. We define the concept of an equilibrium state in this dynamic game and present iterative algorithms that create feasible trajectories tending to equilibrium states under rather general conditions.
\end{abstract}

Key words: Dynamic games, discrete time, incomplete knowledge, utility shares distributions, equilibrium states, solution trajectories.

\section{Introduction}

The usual approach to formulation of various decision making problems is to choose the best variant under the current knowledge about the problem under solution. This means that all the necessary knowledge about the problem data and all the parameters should be derived beforehand. In this situation, the solution of the basic problem can be treated as absolute. This approach may admit proper corrections caused by possible data perturbations, but the model remains the same in general. At the same time, the presentation of all the model functions, constraints, and parameters may vary together with changes of the real system states. Moreover, only some limited information about the system may be known at each state. Therefore, our decisions will be then dependent on the current state and this fact should be reflected in the mathematical formulation of the decision making problem.

Recently, a new approach to optimization formulations of decision making problems was proposed in [1] where they are treated as relative or subjective optimization problems with respect to system states and give (quasi-)equilibrium problems. In [2], a rather general class of relative optimization problems in metric spaces was presented. It was shown that the quasi-equilibrium type formulations of relative optimization

\footnotetext{
${ }^{1}$ Department of System Analysis and Information Technologies, Kazan Federal University, ul. Kremlevskaya, 18, Kazan 420008, Russia.

E-mail: konn-igor@ya.ru
} 
problems admit rather simple descent solution methods and give suitable trajectories tending to a relatively optimal state.

In this paper, we apply the above approach to game problems. The basic model represents a discrete time dynamic system with active elements (players). Each state of the system is associated with the common system utility value and shares for all the players. Feasible coalitions of the players can change system states, but each move requires certain expenses. The players may have only restricted and local knowledge about the system. We define the concept of an equilibrium state in this dynamic game and propose a simple descent type solution method for creating suitable trajectories converging to an equilibrium state under mild conditions.

\section{Basic Problem Formulations}

We describe a general model of a system whose possible states are contained in a set $X \subseteq E$ where $E$ is a metric space. The system involves $m$ active elements (players). Let $M=\{1, \ldots, m\}$ and let $\mathcal{M} \subseteq \Pi(M)$ denote the set of feasible coalitions that can change system states. Here and below $\Pi(F)$ denotes the family of all nonempty subsets of a set $F$. Each state $x \in X$ is associated with the common system utility value $\varphi(x)$ and a set of player shares

$$
A(x) \subseteq S_{+}^{m}=\left\{u \in \mathbb{R}^{m} \mid \sum_{i=1}^{m} u_{i}=1, u_{i} \geq 0, i=1, \ldots, m\right\} .
$$

This means that the set-valued shares mapping $x \mapsto A(x)$ is defined on $X$. The actual vector of player shares $a(x) \in A(x)$ becomes known only after arrival to the state $x$. Each feasible coalition $I \in \mathcal{M}$ can define its set of feasible states $D_{I}(x)$ at state $x$ in the sense that this coalition $I$ can move the system from $x$ to $y$ and evaluate the common system utility value $\varphi(y)$ if $y \in D_{I}(x)$. Next, each move $(x \rightarrow y)$ accomplished by coalition $I$ requires its common expenses $c_{I}(x, y)$. We suppose that $c_{I}(x, y)$ is nonnegative and known at $x$ to any player of this coalition for any $y \in D_{I}(x)$. Hence, we can define the estimate of pure expenses of coalition $I$ for the move $(x \rightarrow y)$ as follows

$$
f_{I}(x, y)=a_{I}(x)[\varphi(x)-\varphi(y)]+c_{I}(x, y)
$$

where

$$
a_{I}(x)=\sum_{i \in I} a_{i}(x)
$$

This means all the players of any active coalition agree to bear the expenses related to the corresponding system move. Then we can define the following equilibrium problem.

Problem (P1) Find a point $x^{*} \in X$ such that

$$
f_{I}\left(x^{*}, y\right) \geq 0 \quad \forall y \in D_{I}\left(x^{*}\right), \forall I \in \mathcal{M} .
$$


Therefore, if $x^{*}$ is an equilibrium state in the sense of (1), no active coalition has a positive profit estimate for any feasible move from this state. Solution of Problem (P1) may be found by an iterative sequence with non-negative profit estimates. The starting state $x^{0} \in X$ is supposed to be known. We will say that a sequence $\left\{x^{k}\right\} \subset X$ is a feasible trajectory if $x^{k+1} \in D_{I}\left(x^{k}\right)$ and $I \in \mathcal{M}$ for each number $k$. Then we can define the dynamic equilibrium problem.

Problem (P2) Find a feasible trajectory $\left\{x^{k}\right\}$ with the initial state $x^{0} \in X$ and negative pure move expenses estimates for the corresponding active coalitions such that it either terminates at a solution of Problem $(\mathbf{P} 1)$ or its limit points are solutions of Problem (P1).

The above framework is rather general and admits various implementation rules. For instance, many rules can be taken for distribution of coalition move expenses among the players. However, any rule should provide positive personal profit estimates if so is the common coalition profit estimate. For instance, if $f_{I}(x, y)<0$, we can define the $i$-th player personal profit estimate

$$
\mu_{I}^{i}(x, y)=a_{i}(x)[\varphi(y)-\varphi(x)]-a_{i}(x) c_{I}(x, y) / a_{I}(x)=-a_{i}(x) f_{I}(x, y) / a_{I}(x)>0
$$

if $a_{i}(x)>0$. Clearly, coalition $I$ may be profitable for the move $(x \rightarrow y)$ if

$$
c_{I}(x, y)<\sum_{i \in I} c_{i}(x, y)
$$

especially for any player $j$ such that $a_{j}(x)=0$. In general, if $I^{\prime \prime}=I^{\prime} \bigcup\{j\}$ and $c_{I^{\prime \prime}}(x, y)<c_{I^{\prime}}(x, y)$, then coalition $I^{\prime \prime}$ is more profitable for all the players from coalition $I^{\prime}$ with respect to move $(x \rightarrow y)$ under the same cost distribution rule.

It should be observed that the family $\mathcal{M}$ determines the kind of the presented game model. In fact, if $\mathcal{M}=(\{1\}, \ldots,\{m\})$, we have a non-cooperative game, whereas $\mathcal{M}=\Pi(M)$ together with (2) corresponds to a complete cooperative game setting. At the same time, the players can also behave in a non-cooperative manner if (2) does not hold, i.e. then

$$
c_{I}(x, y) \geq \sum_{i \in I} c_{i}(x, y)
$$

The set-valued mappings $x \mapsto D_{I}(x)$ enable us to determine all the gaming restrictions. It seems rather natural to suppose that $x \in D_{I}(x)$ for any $x \in X$ and $I \in \mathcal{M}$, but this condition is not obligatory. Next, setting $D_{I}(x)=\{x\}$ means that coalition $I$ can not change the system state $x$. The choice of the set-valued shares mapping $x \mapsto A(x)$ is also determined by the rules of the game under consideration. For example, they can involve the condition

$$
a_{I}(x) \leq a_{I}(y) \text { if } y \in D_{I}(x)
$$


or

$$
a_{I}(y)=\arg \max \left\{a_{I} \mid a \in A(y)\right\} .
$$

We observe that a solution of the global optimization problem

$$
\max _{x \in X} \rightarrow \varphi(x)
$$

is always a solution of Problem (P1) in this setting, but Problem (P1) may have other solutions as the following simple examples illustrate.

Example 1 Let $X=[0,1]$ and $\varphi(x)=x+1$, then the point $x^{*}=1$ is a unique solution of (3). Next, let $M=\{1,2\}, \mathcal{M}=(\{1\},\{2\},\{1,2\}), a(x) \equiv(0.5,0.5)^{\top}$, $\left.D_{I}(x)=[x-0.1, x+0.1)\right] \bigcap X$ for any $I \in \mathcal{M}$, and $x^{0}=0$. Also, set $c_{I}(x, y)=$ $2(x-y)^{2}+8 x|x-y|$ for $I=\{1,2\}$ and $c_{I}(x, y)=3(x-y)^{2}+10 x|x-y|$ for $I=\{1\}$ and $I=\{2\}$. Then the cooperative behaviour is more reasonable for both the players and we fix $I=\{1,2\}$. Take the first move $\left(x^{0} \rightarrow x^{1}\right)$ where $x^{1}=x^{0}+0.1=0.1$, then we have the estimate

$$
f_{I}\left(x^{0}, x^{1}\right)=-0.1+0.02<0,
$$

i.e. it is profitable. However, this is not the case for the next similar move $\left(x^{1} \rightarrow x^{\prime}\right)$ where $x^{\prime}=x^{1}+0.1=0.2$ since

$$
f_{I}\left(x^{1}, x^{\prime}\right)=-0.1+0.02+0.08=0 .
$$

We take the next move $\left(x^{1} \rightarrow x^{2}\right)$ with the reduced step and choose $x^{2}=x^{1}+0.05=$ 0.15. It is profitable since

$$
f_{I}\left(x^{1}, x^{2}\right)=-0.05+0.005+0.04=-0.005<0 .
$$

It appears that $x^{2}$ is a solution of Problem (P1) since

$$
f_{I}\left(x^{2}, x\right) \geq 0 \quad \forall x \in D_{I}\left(x^{2}\right) .
$$

The trajectory $\left\{x^{0}, x^{1}, x^{2}\right\}$ is feasible, therefore, it is a solution of Problem (P2).

The next example also shows that solutions may depend on trajectories.

Example 2 We take a system with a finite number of states, namely, we set $X=$ $\left\{v^{1}, v^{2}, v^{3}, v^{4}\right\}, \varphi\left(v^{1}\right)=10, \varphi\left(v^{2}\right)=\varphi\left(v^{3}\right)=11, \varphi\left(v^{4}\right)=12$; see Figure 1 . The arcs indicate feasible profitable moves. Also, we can determine the distance $d(v, v)=0$ for any $v \in X, d(u, v)=1$ for any pair of different vertices $u, v \in X$ joined by one arc, and $d(u, v)=2$ for any other different vertices $u, v \in X$. Then the set $X$ is a metric space. Next, let $M=\{1,2\}, \mathcal{M}=(\{1\},\{2\})$, hence the players will behave in a non-cooperative manner. We define their state shares as follows:

$$
a\left(v^{1}\right)=(0.5,0.5)^{\top}, a\left(v^{2}\right)=(0.9,0.1)^{\top}, a\left(v^{3}\right)=a\left(v^{4}\right)=(0.1,0.9)^{\top},
$$


and the feasible sets:

$$
\begin{aligned}
& D_{\{1\}}\left(v^{1}\right)=D_{\{2\}}\left(v^{1}\right)=\left\{v^{2}, v^{3}\right\} \\
& D_{\{1\}}\left(v^{2}\right)=D_{\{2\}}\left(v^{2}\right)=\left\{v^{4}\right\}, D_{\{1\}}\left(v^{3}\right)=D_{\{2\}}\left(v^{3}\right)=\left\{v^{4}\right\} .
\end{aligned}
$$

Next, we define the move expenses as follows:

$$
\begin{aligned}
& c_{\{1\}}\left(v^{1}, v^{2}\right)=c_{\{2\}}\left(v^{1}, v^{3}\right)=0.25, c_{\{1\}}\left(v^{1}, v^{3}\right)=c_{\{2\}}\left(v^{1}, v^{2}\right)=1, c_{\{2\}}\left(v^{2}, v^{4}\right)=0.5, \\
& c_{\{1\}}\left(v^{2}, v^{4}\right)=c_{\{2\}}\left(v^{3}, v^{4}\right)=1, c_{\{1\}}\left(v^{3}, v^{4}\right)=0.2 .
\end{aligned}
$$

We denote by $p_{i}(x)$ the pure profit of player $i$ at system state $x$, which is equal to his/her profit at $x$ minus the trajectory expenses. If $x^{0}=v^{1}$, then $p_{1}\left(x^{0}\right)=p_{2}\left(x^{0}\right)=5$. However, $x^{0}$ is not a solution of Problem (P1).

Let us take the first move $\left(v^{1} \rightarrow v^{2}\right)$. Then we have the estimates

$$
f_{\{1\}}\left(v^{1}, v^{2}\right)=-0.5+0.25<0 \text { and } f_{\{2\}}\left(v^{1}, v^{2}\right)=-0.5+1>0,
$$

i.e. the move may be profitable for the first player. Hence, we can check the second move $\left(v^{2} \rightarrow v^{4}\right)$. We have the estimates

$$
f_{\{1\}}\left(v^{2}, v^{4}\right)=-0.9+1>0 \text { and } f_{\{2\}}\left(v^{2}, v^{4}\right)=-0.1+0.05<0,
$$

i.e. the move may be profitable for the second player. Therefore, $v^{4}$ is a solution of Problem (P1) and the trajectory $\left\{v^{1}, v^{2}, v^{4}\right\}$ is a solution of Problem (P2). Besides, $v^{4}$ is a solution of $(3)$. Let us compare the pure profits of the players:

$$
p_{1}\left(v^{2}\right)=9.65>5, p_{2}\left(v^{2}\right)=1.1<5, p_{1}\left(v^{4}\right)=0.95<5, p_{2}\left(v^{4}\right)=10.75>5 \text {. }
$$

Let us take the other first move $\left(v^{1} \rightarrow v^{3}\right)$. Then we have the estimates

$$
f_{\{1\}}\left(v^{1}, v^{3}\right)=-0.5+1>0 \text { and } f_{\{2\}}\left(v^{1}, v^{3}\right)=-0.5+0.25<0,
$$

i.e. the move may be profitable for the second player. Hence, we can check the second move $\left(v^{3} \rightarrow v^{4}\right)$. We have the estimates

$$
f_{\{1\}}\left(v^{3}, v^{4}\right)=-0.1+0.2>0 \text { and } f_{\{2\}}\left(v^{3}, v^{4}\right)=-0.9+1>0,
$$

i.e. they are not profitable. Therefore, $v^{3}$ is a solution of Problem (P1) and the trajectory $\left\{v^{1}, v^{3}\right\}$ is a solution of Problem (P2). Let us compare the pure profits of the players:

$$
p_{1}\left(v^{3}\right)=1.1<5, p_{2}\left(v^{3}\right)=9.65>5
$$




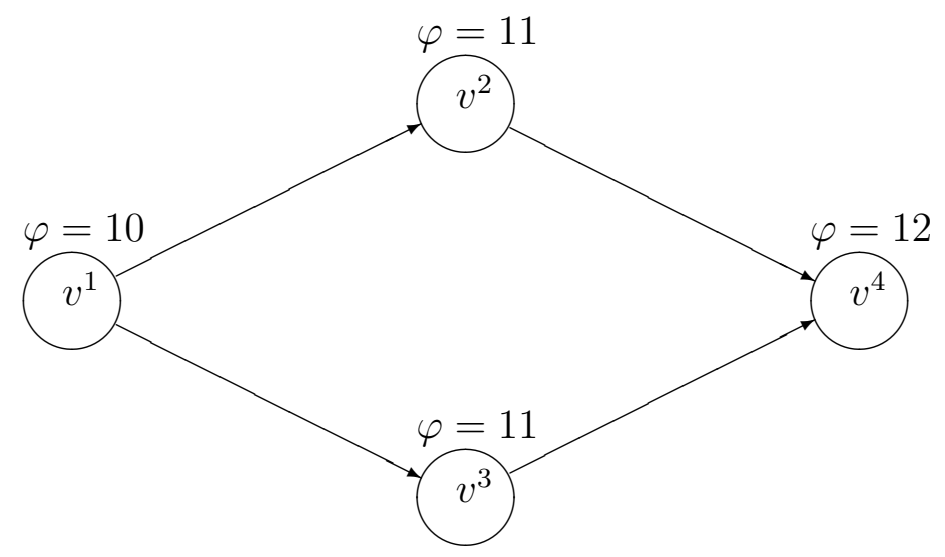

Figure 1: An equilibrium problem on a graph

\section{The Basic Method and Its Convergence}

We will use the following set of basic assumptions.

(A1) The set $X \subseteq E$ is nonempty and closed, the function $\varphi: X \rightarrow \mathbb{R}$ is continuous, for each $I \in \mathcal{M}$ the bi-function $c_{I}: X \times X \rightarrow \mathbb{R}$ is non-negative and continuous.

(A2) For some number $\alpha \leq u\left(x^{0}\right)$ the set

$$
X_{\alpha}=\{x \in X \mid \varphi(x) \geq \alpha\}
$$

is compact.

(A3) For each $I \in \mathcal{M}$ the mapping $D_{I}: X \rightarrow \Pi(X)$ is lower semi-continuous on $X$.

(A4) The mapping $A: X \rightarrow \Pi\left(S_{+}^{m}\right)$ is upper semi-continuous on $X$ and has closed values on $X$.

We recall that a set-valued mapping $T: X \rightarrow \Pi(X)$ is said to be

(a) upper semicontinuous on $X$, if for each point $v \in X$ and for each open set $U$ such that $U \supseteq T(v)$, there is an open neighborhood $\tilde{V}$ of $v$ such that $T(w) \subset U$ whenever $w \in \tilde{V} \cap X$;

(b) lower semicontinuous on $X$, if for each point $v \in X$ and for each open set $U$ such that $U \bigcap T(v) \neq \varnothing$, there is an open neighborhood $\tilde{V}$ of $v$ such that $U \bigcap T(w) \neq \varnothing$ whenever $w \in \tilde{V} \cap X$.

Clearly, (A2) is a general coercivity condition, which together with continuity of $\varphi$ implies that the global optimization problem (3) has a solution and that

$$
\varphi^{*}=\sup _{x \in X} \varphi(x)<+\infty .
$$

Hence, Problem (P1) also has a solution. The solutions of both (P1) and (P2) can be found by the following threshold descent method (TDM). 


\section{Method (TDM).}

Initialization: Take the given point $x^{0}$, choose a sequence $\left\{\delta_{l}\right\} \searrow 0$. Set $l=1, k=0$, $z^{0}=x^{0}$.

Step 1: Given a point $z^{k} \in X$ and a vector $a\left(z^{k}\right) \in A\left(z^{k}\right)$, find a coalition $J=$ $J(k) \in \mathcal{M}$ such that

$$
\exists z^{k+1} \in D_{J}\left(z^{k}\right), \quad f_{J}\left(z^{k}, z^{k+1}\right)<-\delta_{l},
$$

set $k=k+1$ and go to the beginning of Step 1. Otherwise, i.e., if this coalition does not exist, go to Step 2.

Step 2: Set $x^{l}=z^{k}, l=l+1$ and go to Step 1 .

Therefore, $\delta_{l}$ stands for the current descent threshold, which determines the sufficient profit for the movement. The index $l$ is a counter for the number of restarts (threshold changes). The choice of $z^{k+1}$ in accordance with (5) depends on peculiarities of the problem. In particular, it can be based on a solution of the auxiliary problem

$$
\min _{z \in D_{J}\left(z^{k}\right)} \rightarrow\left\{c_{J}\left(z^{k}, z\right)-a_{J}\left(z^{k}\right) \varphi(z)\right\}
$$

Theorem 1 Let assumptions (A1)-(A4) be fulfilled. Then the sequence $\left\{x^{l}\right\}$ generated by Method (TDM) has limit points, all these limit points are solutions of Problem (P1), and the sequence $\left\{z^{k}\right\}$ solves Problem (P2).

Proof. The assertion will be proved in several steps.

Step 1: For each fixed $k$ relation (5) implies

$$
a_{J(k)}\left(z^{k}\right)>0
$$

and

$$
\varphi\left(z^{k+1}\right)-\varphi\left(z^{k}\right)>\delta_{l}
$$

If (7) does not hold (5) gives

$$
0 \leq c_{J(k)}\left(z^{k}, z^{k+1}\right)<-\delta_{l}<0,
$$

which is a contradiction. Next, by definition,

$$
\begin{aligned}
\varphi\left(z^{k+1}\right)-\varphi\left(z^{k}\right) & =\left[c_{J(k)}\left(z^{k}, z^{k+1}\right)-f_{J(k)}\left(z^{k}, z^{k+1}\right)\right] / a_{J(k)}\left(z^{k}\right) \\
& >\left[c_{J(k)}\left(z^{k}, z^{k+1}\right)+\delta_{l}\right] / a_{J(k)}\left(z^{k}\right) \geq \delta_{l} .
\end{aligned}
$$

Step 2: For each $l$ the number of changes of the index $k$ is finite.

The assertion follows from (8) and (A2).

Step 3: The sequence $\left\{x^{l}\right\}$ has limit points, all these limit points are solutions of Problem (P1).

From Steps 1-2 it follows that the sequence $\left\{x^{l}\right\}$ is infinite and is contained in the 
compact set $X_{\alpha}$ due to (A2). It follows that $\left\{x^{l}\right\}$ has limit points. For each $l$ from the definition we have

$$
a\left(x^{l}\right) \in A\left(x^{l}\right), \quad f_{I}\left(x^{l}, y\right) \geq-\delta_{l} \quad \forall y \in D_{I}\left(x^{l}\right), \forall I \in \mathcal{M} .
$$

Let $\bar{x}$ be an arbitrary limit point of $\left\{x^{l}\right\}$, i.e. $\left\{x^{l_{s}}\right\} \rightarrow \bar{x}$. Then $\bar{x} \in X$ since $X$ is closed. Since the sequence $\left\{a\left(x^{l_{s}}\right)\right\}$ is bounded, it has limit points. Without loss of generality we can suppose that

$$
\lim _{s \rightarrow \infty} a\left(x^{l_{s}}\right)=\bar{a}
$$

then $\bar{a} \in A(\bar{x})$ due to (A4).

Take any coalition $I \in \mathcal{M}$ and any $\bar{y} \in D_{I}(\bar{x})$, then there exists a sequence of points $\left\{y^{l_{s}}\right\},\left\{y^{l_{s}}\right\} \rightarrow \bar{y}$ such that $y^{l_{s}} \in D_{I}\left(x^{l_{s}}\right)$ since the mapping $D_{I}$ is lower semi-continuous on $X$ due to (A3). Setting $l=l_{s}$ and $y=y^{l_{s}}$ in (9) and taking the limit $s \rightarrow \infty$ give

$$
\bar{a} \in A(\bar{x}), \quad f_{I}(\bar{x}, \bar{y}) \geq 0,
$$

i.e. $\bar{x}$ is a solution of Problem (P1). It follows that $\left\{z^{k}\right\}$ is a solution of Problem (P2).

The assumptions of Theorem 1 can be modified in a complete metric space setting. In particular, we can then remove the compactness assumption.

(B1) The set $X \subseteq E$ is nonempty and closed, $E$ is a complete metric space with the metric bi-function $d: X \times X \rightarrow \mathbb{R}$, the function $\varphi: X \rightarrow \mathbb{R}$ is continuous, relation (4) holds.

(B2) For each $I \in \mathcal{M}$ the bi-function $c_{I}: X \times X \rightarrow \mathbb{R}$ is non-negative and continuous, there exists an increasing continuous function $\theta: \mathbb{R} \rightarrow \mathbb{R}$ such that $\theta(0)=0$ and that for all $x, y \in X$ we have $\theta[d(x, y)] \leq c_{I}(x, y)$ for any $I \in \mathcal{M}$.

(B3) The cost bi-functions $c_{I}: X \times X \rightarrow \mathbb{R}, I \in \mathcal{M}$ satisfy the coalitional triangle inequality, i.e., for any $I, J \in \mathcal{M}$ and for any $x, y, z \in X$ there exists $K \in \mathcal{M}$ such that

$$
c_{I}(x, z)+c_{J}(z, y) \geq c_{K}(x, y)
$$

Theorem 2 Let assumptions (B1)-(B3) and (A3)-(A4) be fulfilled. Then the sequence $\left\{x^{l}\right\}$ generated by Method (TDM) converges to a solution of Problem (P1), and the sequence $\left\{z^{k}\right\}$ solves Problem (P2).

Proof. The assertion will be proved in several steps.

Step 1: For each fixed $k$ relation (5) implies (7) and (8).

This is proved as in Step 1 of Theorem 1.

Step 2: For each $l$ the number of changes of the index $k$ is finite.

The assertion follows from (8) and (B1). 
Step 3: The sequence $\left\{z^{k}\right\}$ converges to a point $\bar{x} \in X$.

It also follows from (8) and (B1) that

$$
\lim _{k \rightarrow \infty} \varphi\left(z^{k}\right)=\tilde{\varphi}<+\infty
$$

besides, (5) now gives

$$
c_{J(k)}\left(z^{k}, z^{k+1}\right)<a_{J(k)}\left(z^{k}\right)\left[\varphi\left(z^{k+1}\right)-\varphi\left(z^{k}\right)\right] \leq \varphi\left(z^{k+1}\right)-\varphi\left(z^{k}\right) .
$$

Take any indices $k$ and $m=k+p$, then we have

$$
\begin{aligned}
\theta\left[d\left(z^{k}, z^{k+p}\right)\right] & \leq c_{I(k)}\left(z^{k}, z^{k+p}\right) \leq c_{J(k)}\left(z^{k}, z^{k+1}\right)+\ldots+c_{J(k+p-1)}\left(z^{k+p-1}, z^{k+p}\right) \\
& \leq \varphi\left(z^{k+p}\right)-\varphi\left(z^{k}\right)
\end{aligned}
$$

for some $I(k) \in \mathcal{M}$ due to (B2) and (B3). On account of (10) we now obtain that for any number $\alpha>0$ there exists an index $k^{\prime}$ such that $d\left(z^{k}, z^{m}\right)<\alpha$ if $\min \{k, m\}=$ $k>k^{\prime}$. Hence, $\left\{z^{k}\right\}$ is a Cauchy sequence and it converges to a point $\bar{x} \in X$ since $X$ is closed.

Step 4: The sequence $\left\{x^{l}\right\}$ converges to a point $\bar{x} \in X$, which is a solution of Problem (P1).

Since the sequence $\left\{x^{l}\right\}$ is contained in $\left\{z^{k}\right\}$ and is infinite due to Step 2, Step 3 implies that $\left\{x^{l}\right\}$ converges to the point $\bar{x} \in X$. The rest part of the proof is the same as in Step 3 of Theorem 1.

Remark 1 Let us replace condition (A3) with the following.

$\left(\mathbf{A} 3^{\prime}\right)$ For each $I \in \mathcal{M}$ the mapping $D_{I}: X \rightarrow \Pi(X)$ is lower semi-continuous on $X$ and $x \in D_{I}(x)$ for any $x \in X$.

Similarly, we can take the somewhat stronger formulations of Problems (P1) and (P2).

Problem (P1') Find a point $x^{*} \in X$ such that

$$
x^{*} \in D_{I}\left(x^{*}\right), f_{I}\left(x^{*}, y\right) \geq 0 \quad \forall y \in D_{I}\left(x^{*}\right), \forall I \in \mathcal{M} .
$$

Problem (P2') Find a feasible trajectory $\left\{x^{k}\right\}$ with the initial state $x^{0} \in X$ and negative pure move expenses estimates for the corresponding active coalitions such that it either terminates at a solution of Problem $\left(\mathbf{P} \mathbf{1}^{\prime}\right)$ or its limit points are solutions of Problem $\left(\mathbf{P} 1^{\prime}\right)$.

Then the assertions of Theorems 1 and 2 remain true where $(\mathbf{A} 3),(\mathbf{P} 1)$, and (P2) are replaced with $\left(\mathbf{A} 3^{\prime}\right),\left(\mathbf{P} \mathbf{1}^{\prime}\right)$, and $\left(\mathbf{P} \mathbf{2}^{\prime}\right)$, respectively. 


\section{The Simple Descent Method}

Let us now take the simple descent method (SDM) for Problems (P1) and (P2), which does not involve any threshold.

Method (SDM).

Initialization: Take the given point $x^{0} \in X$, set $k=0$.

Step 1: Given a point $x^{k} \in X$ and a vector $a\left(x^{k}\right) \in A\left(x^{k}\right)$, find a coalition $J=$ $J(k) \in \mathcal{M}$ such that

$$
\exists x^{k+1} \in D_{J}\left(x^{k}\right), \quad f_{J}\left(x^{k}, x^{k+1}\right)<0,
$$

set $k=k+1$ and go to the beginning of Step 1. Otherwise, i.e., if this coalition does not exist, stop.

However, it does not converge to a solution under the assumptions of the previous section even in case $m=1$; see e.g. [2, Example 4.1]. However, (SDM) can be useful in the case when there exists a lower positive threshold for move expenses. Then we can relax essentially the other assumptions.

(C1) The set $X \subseteq E$ is nonempty, relation (4) holds. The mapping $A: X \rightarrow \Pi\left(S_{+}^{m}\right)$ has non-empty values on $X$.

(C2) For each $I \in \mathcal{M}$ the bi-function $c_{I}: X \times X \rightarrow \mathbb{R}$ is non-negative, there exists a number $\delta>0$ such that $c_{I}(x, y) \geq \delta$ for all $x, y \in X, x \neq y$ and for any $I \in \mathcal{M}$.

(C3) For each $I \in \mathcal{M}$ the mapping $D_{I}: X \rightarrow \Pi(X)$ has non-empty values on $X$.

We can thus remove all the continuity and compactness assumptions. Then, Method (SDM) solves both the problems in a finite number of iterations, as the following theorem states.

Theorem 3 Let assumptions (C1)-(C3) be fulfilled. Then the sequence $\left\{x^{k}\right\}$ generated by Method (SDM) solves Problem (P2). It is finite and stops at a solution of Problem (P1).

Proof. It suffices to prove the finiteness of Method (SDM). For each $k$ relation (12) implies $a_{J(k)}\left(x^{k}\right)>0$. In fact, otherwise we have

$$
0 \leq c_{J(k)}\left(x^{k}, x^{k+1}\right)<0
$$

which is a contradiction. Next, by definition,

$$
\begin{aligned}
\varphi\left(x^{k+1}\right)-\varphi\left(x^{k}\right) & =\left[c_{J(k)}\left(x^{k}, x^{k+1}\right)-f_{J(k)}\left(x^{k}, x^{k+1}\right)\right] / a_{J(k)}\left(x^{k}\right) \\
& >c_{J(k)}\left(z^{k}, z^{k+1}\right) \geq \delta>0 .
\end{aligned}
$$

If the sequence $\left\{x^{k}\right\}$ is infinite, the above inequality implies $\varphi\left(x^{k}\right) \rightarrow+\infty$ as $k \rightarrow \infty$, which contradicts (4). 
Remark 2 As in Remark 1, we can replace condition (C3) with the following. (C3') For each $I \in \mathcal{M}$ it holds that $x \in D_{I}(x)$ for any $x \in X$.

Similarly, we can take the formulations $\left(\mathbf{P} 1^{\prime}\right)$ and $\left(\mathbf{P} \mathbf{2}^{\prime}\right)$ instead of $(\mathbf{P} 1)$ and $(\mathbf{P} 2)$. Then the assertion of Theorem 3 remains true where $(\mathbf{C} 3),(\mathbf{P} 1)$, and (P2) are replaced with $\left(\mathbf{C} 3^{\prime}\right),\left(\mathbf{P} 1^{\prime}\right)$, and $\left(\mathbf{P} 2^{\prime}\right)$, respectively.

\section{$5 \quad$ Examples of Models}

We now describe some applied models, which can be formulated within the proposed framework.

Example 3 (Dynamic oligopoly with coalitions). In the classical oligopoly model (see e.g. [3]), it is assumed that there are $m$ industrial firms supplying a homogeneous commodity and that the price $p$ depends on its total quantity $x$, i.e. $p=p(x)$. Next, the value $h_{i}\left(z_{i}\right)$ represents the $i$-th firm individual expenses of supplying $z_{i}$ units of the product. It follows that

$$
x=x(z)=\sum_{i=1}^{m} z_{i},
$$

where $z=\left(z_{1}, z_{2}, \ldots, z_{m}\right)^{\top}$. In such a way, we obtain a noncooperative static game where the $i$-th player (firm) has its particular strategy set $\mathbb{R}_{+}$and a payoff (profit) function

$$
\mu_{i}(z)=z_{i} p(x(z))-h_{i}\left(z_{i}\right)
$$

$i=1, \ldots, m$.

It is natural to suppose that the firms may create coalitions in order to reduce their common production transition expenses. Then we can define the model of the system whose states are determined by the total supply quantity $x$, so that $X=[0, b]$ gives the set of all the feasible states, and define the common system utility (profit) function $\varphi: X \rightarrow \mathbb{R}$, which is also supposed to be non-decreasing. In general, $\varphi(x)$ may involve industrial and pollution treatment expenses at the production level $x$. For the sake of simplicity, we take the fixed value of the player shares set

$$
A(x) \equiv S_{+}^{m},
$$

then some chosen $a(x) \in A(x)$ gives the $i$-th individual utility $\varphi_{i}(x)=a_{i}(x) \varphi(x)$ at $x$.

As above, we choose the set of feasible coalitions $\mathcal{M} \subseteq \Pi(M)$ that can change system states. For each coalition $I \in \mathcal{M}$ we define the set of feasible states $D_{I}(x)=$ $\left[x-\varepsilon_{I}, x+\varepsilon_{I}\right] \cap X$ at state $x \in X$ where $\varepsilon_{I}>0$. Also, each move $(x \rightarrow y)$ accomplished by coalition $I$ implies its common transition expenses $c_{I}(x, y) \geq 0$, but the move estimate must be profitable, i.e.,

$$
f_{I}(x, y)=a_{I}(x)[\varphi(x)-\varphi(y)]+c_{I}(x, y)<0 .
$$


The actual vector of player shares $a(y)$ is determined by coalition $I$ after arrival to the state $y$, where one of the suitable distribution rules or their combination can be taken. For instance, the individual utilities can be first calculated as follows:

$$
\varphi_{i}(y)=a_{i}(x) \varphi(y) \text { if } i \notin I \text { and } \varphi_{i}(y)=\varphi_{i}(x)+q_{i}[\varphi(y)-\varphi(x)] \text { if } i \in I,
$$

where $q_{i} \in(0,1)$ is a chosen parameter. It is possible to take $q_{i}=1 /|I|$ where $|I|$ is the number of elements in $I$, or set $q_{i}=a_{i}(x) / a_{I}(x)$. Then we can set $a_{i}(y)=\varphi_{i}(y) / \varphi(y)$ for $i=1, \ldots, m$. We can now formulate Problems (P1) and (P2) that determine relative equilibrium states and trajectories of the system. The pure profits will depend on the movement trajectory. If the function $\varphi$ is continuous, and for each $I \in \mathcal{M}$ the bi-function $c_{I}: X \times X \rightarrow \mathbb{R}$ is continuous, then assumptions (A1)-(A4) are fulfilled, and we can apply Method (TDM). Clearly, this approach can be easily extended to the multi-commodity case.

Example 4 (Resource allocation in telecommunication networks). We first describe an optimal flow distribution problem in telecommunication networks; see e.g. [4]. The network contains $n$ transmission links (arcs) and accomplishes some submitted data transmission requirements from $n$ selected pairs of origin-destination vertices within a fixed time period. Denote by $u_{i}$ and $d_{i}$ the current and maximal value of data transmission for pair demand $i$, respectively, and by $x_{j}$ the current network capacity of link $j$. Each pair demand is associated with a unique data transmission path, hence each link $j$ is associated uniquely with the set $N(j)$ of pairs of origin-destination vertices, whose transmission paths contain this link. For each pair demand $i$ we denote by $h_{i}\left(u_{i}\right)$ the network profit value at the data transmission volume $u_{i}$. Then we can write the network income maximization problem as follows:

$$
\max \rightarrow \sum_{i=1}^{m} h_{i}\left(u_{i}\right)
$$

subject to

$$
\begin{aligned}
& \sum_{i \in N(j)} u_{i} \leq x_{j}, j=1, \ldots, n \\
& 0 \leq u_{i} \leq d_{i}, i=1, \ldots, m .
\end{aligned}
$$

Denote by $\alpha(x)$ the optimal value of problem (13)-(15) depending on the right-hand sides $x$ of the constraints as parameters. Also, let $\beta(x)$ denote the network facility maintenance expenses at the capacity vector $x$. Then we can define the network utility (profit) function value

$$
\varphi(x)=\alpha(x)-\beta(x)
$$

Let $X$ denote the set of all the feasible capacity profiles, for instance, we can take

$$
X=\left\{x \in \mathbb{R}^{n} \mid 0 \leq x_{j} \leq b_{j}, j=1, \ldots, n\right\} .
$$


That is, $X$ stands for the set of feasible states of the system. Here $b_{j}$ denotes the maximal capacity of link $j$.

However, it is natural to suppose that each link can be served by different telecommunication providers (see e.g. [5]), which leads to a more general setting of the choice of the link capacities. Hence, the providers may create coalitions in order to reduce their common capacity transition expenses. More precisely, it is assumed that there are $m$ providers (players), they can supply telecommunication services to the users. For the sake of simplicity, we again take the fixed value of the player shares set

$$
A(x) \equiv S_{+}^{m},
$$

then some chosen $a(x) \in A(x)$ gives the $i$-th individual utility $\varphi_{i}(x)=a_{i}(x) \varphi(x)$ at $x \in X$.

As above, we choose the set of feasible coalitions $\mathcal{M} \subseteq \Pi(M)$ that can change system states. For each coalition $I \in \mathcal{M}$ we define the set of feasible states $D_{I}(x)=$ $\left.\left[x-\varepsilon_{I} \mathbf{e}, x+\varepsilon_{I} \mathbf{e}\right)\right] \bigcap X$ at state $x \in X$ where $\mathbf{e}=(1, \ldots, 1)^{\top} \in \mathbb{R}^{m}$ and $\varepsilon_{I}>0$. Next, each move $(x \rightarrow y)$ accomplished by coalition $I$ implies its common capacity transition expenses $c_{I}(x, y) \geq 0$. This requires for the move estimate to be profitable, i.e.,

$$
f_{I}(x, y)=a_{I}(x)[\varphi(x)-\varphi(y)]+c_{I}(x, y)<0 .
$$

The actual vector of player shares $a(y)$ is also determined by coalition $I$ after arrival to the state $y$, where a suitable distribution rule can be taken. Some of these distribution rules are given in Example 3. We can formulate Problems (P1) and (P2) that determine equilibrium allocations and trajectories of the system. The pure profits will depend on the movement trajectory. If the function $\varphi$ is continuous, and for each $I \in \mathcal{M}$ the bi-function $c_{I}: X \times X \rightarrow \mathbb{R}$ is continuous, then assumptions (A1)-(A4) are fulfilled, and we can apply Method (TDM).

\section{References}

[1] Konnov, I.V. (2019) Equilibrium formulations of relative optimization problems. Mathem. Meth. Oper. Res. 90, 137-152

[2] Konnov, I.V. (2021) A general class of relative optimization problems. Mathem. Meth. Oper. Res. https://doi.org/10.1007/s00186-021-00741-1

[3] Okuguchi, K. and Szidarovszky, F. (1990) The Theory of Oligopoly with Multiproduct Firms, Springer-Verlag, Berlin

[4] Kelly, F.P., Maulloo, A., Tan, D. (1998) Rate control for communication networks: shadow prices, proportional fairness and stability. J. Oper. Res. Soc. 49, 237-252

[5] Alpcan T., Boche H., Honig M., Poor H.V., Eds. (2014) Mechanisms and Games for Dynamic Spectrum Allocation, Cambridge University Press, New York 